\title{
EFEITO DA IMERSÃO EM ÁGUA FRIA SOBRE A RECUPERAÇÃO PÓS-ESFORÇO EM ATLETAS DE JIU-JITSU
}

\author{
EFFECT OF COLD WATER IMMERSION ON POST-EXERTION RECOVERY IN JUU-JITSU ATHLETES
}

ARTIGO ORIGINAL

EFECTO DE LA INMERSIÓN EN AGUA FRÍA SOBRE LA RECUPERACIÓN POST-ESFUERZO EN

Original ARTICLE

Artículo Original

\section{ATLETAS DE JIU-JITSU}

Paulo Roberto Gonçalves Silva' (Profissional de Educação Física) Wonder Passoni Higino ${ }^{2}$ (Profissional de Educação Física) Fabiano Fernandes da Silva² (Profissional de Educação Física) Renato Aparecido de Souza ${ }^{2,3}$ (Fisioterapeuta)

1. Universidade Camilo Castelo Branco (UNICASTELO), Instituto de Engenharia Biomédica, São Paulo, SP, Brasil.

2. Instituto Federal de Educação, Ciência e Tecnologia do Sul de Minas Gerais (IFSULDEMINAS), Campus Muzambinho, MG, Brasil. 3. Universidade Federal de Alfenas (UNIFAL), Programa de Pós-Graduação em Ciências da Reabilitação, MG, Brasil.

\section{Correspondência:}

Renato Aparecido de Souza, IFSULDEMINAS, Campus

Muzambinho. Rua Dinah, 75, Canaã, Muzambinho, Minas Gerais, MG, Brasil. 37890-000.

renato.souza@muz.ifsuldeminas.edu.br

\section{RESUMO}

Introdução: A imersão em água fria (IAF) tem sido usada habitualmente para a recuperação muscular, mesmo com evidências limitadas sobre sua eficácia. Objetivo: O objetivo deste estudo foi investigar os efeitos da IAF depois de uma sessão de treinamento de atletas de jiu-jitsu, sobre creatina quinase (CQ), testes funcionais de força e parâmetros isocinéticos. Métodos: Doze atletas de jiu-jitsu (idade 21,75 $\pm 3,10$ anos; IMC 24,65 $\pm 3,81$ $\mathrm{kg} / \mathrm{m}^{2}$; tempo de treinamento 3,41 $\pm 0,51$ anos) foram recrutados. Considerando que o estudo é cruzado, cada grupo teve a participação dos 12 atletas com wash-out de 30 dias. Os participantes foram divididos em dois grupos experimentais: (I) Grupo controle (CON), no qual os atletas não foram submetidos à IAF e (II) Grupo IAF, no qual os atletas foram submetidos à IAF $\left(\sim 12^{\circ} \mathrm{C}\right)$ durante seis minutos. O esforço físico foi realizado em uma sessão de treino com simulação de lutas de jiu-jitsu, com quatro lutas de cinco minutos cada e intervalo de três minutos entre elas. As variáveis CQ plasmática, protocolo estático e dinâmico do Kimono Grip Strength Test e parâmetros isocinéticos (pico de torque, trabalho e potência) foram avaliadas antes e depois dos procedimentos experimentais de recuperação. Para a análise estatística foi usada a análise de variância de dois fatores (tempo e tratamento). O nível de significância foi de 5\%. Resultados: Observou-se o efeito do tempo ao comparar CQ plasmática (incremento de 174,39 $\pm 99,95 \mathrm{UI} / \mathrm{l}$ para o CON e incremento de 187,91 $\pm 113,02 \mathrm{UI} / \mathrm{I}$ para o IAF) e teste estático do KGST (delta de $-5,83 \pm 9,35 \mathrm{~s}$ para o CON e delta de $-2,83 \pm 13,94 \mathrm{~s}$ para o IAF) antes e depois dos procedimentos experimentais de recuperação, porém, sem efeito do tratamento $(P>0,05)$. Os parâmetros isocinéticos não foram influenciados. Conclusão: Conclui-se que a IAF não promoveu recuperação pós-esforço em atletas de jiu-jitsu. Nível de Evidência Il; ECRC de menor qualidade.

Descritores: Creatina quinase; Crioterapia; Artes marciais; Força muscular; Reabilitação.

\section{ABSTRACT}

Introduction:Cold-water immersion (CWI) has been commonly used for muscle recovery, even with limited evidence of its efficacy. Objective: The aim of this study was to investigate the effects of CWI after a training session of jiu-jitsu athletes on creatine kinase (CK), functional strength tests, and isokinetic parameters. Methods: Twelve jiu-jitsu athletes (age $21.75 \pm 3.10$ years; BMI $24.65 \pm 3.81 \mathrm{~kg} / \mathrm{m}^{2}$; training time $3.41 \pm 0.51$ years) were selected. Considering that this is a crossover study, each group had the participation of 12 athletes with a 30-day wash-out. Participants were divided into two experimental groups: (I) control group (CON), in which the athletes did not underwent CWI, and (II) CWI group, in which athletes underwent $\mathrm{CWI}\left(\sim 12^{\circ} \mathrm{C}\right)$ for six minutes. The physical exertion was carried out in a training session with simulation ofjiu-jitsu fights, with four fights five minutes each and an interval of three minutes between them. Plasma CK, static and dynamic protocols of the Kimono Grip Strength Test, and isokinetic parameters (peaktorque, work, and power) were assessed before and after the experimental recovery procedures. Statistical analysis was performed using two-way analysis of variance (time and treatment). The level of significance was 5\%. Results: The effect of time on the comparison of plasma CK (increase of $174.39 \pm 99.95 \mathrm{IU} / \mathrm{I}$ for CON and increase of $187.91 \pm 113.02 \mathrm{IU} / \mathrm{I}$ for CWI) and static test KGST ( $\triangle t$ of $-5.83 \pm 9.35 \mathrm{~s}$ for $\mathrm{CON}$ and $\Delta t$ of $-2.83 \pm 13.94 \mathrm{~s}$ for $C W I$ ) was observed before and after the experimental recovery procedures, but without treatment effect $(P>0.05)$. Isokinetic parameters were not influenced $(P>0.05)$. Conclusion: It is concluded that the CWI did not promote post-exertion recovery in jiu-jitsu athletes. Level of Evidence ll; Lesser quality RCT.

Keywords: Creatine kinase; Cryotherapy; Martial arts; Muscle strength; Rehabilitation.

\section{RESUMEN}

Introducción: La inmersión en agua fría (IAF) se ha utilizado habitualmente para la recuperación muscular, aun con evidencias limitadas sobre su eficacia. Objetivo: El objetivo de este estudio fue investigar los efectos de la IAF después de una sesión de entrenamiento de atletas dejiu-jitsu, sobre la creatina quinasa (CK), pruebas funcionales de fuerzay parámetros isocinéticos. Métodos: Doce atletas de jiu-jitsu (edad 21,75 $\pm 3,10$ años, IMC 24,65 $\pm 3,81 \mathrm{~kg} / \mathrm{m}^{2}$, tiempo de entrenamiento $3,41 \pm 0,51$ años) fueron reclutados. Considerando que el estudio es cruzado, cada grupo tuvo la participación de los 12 atletas con wash-out de 30 días. Los participantes fueron divididos en dos grupos experimentales: (I) Grupo control (CON), en el cual los atletas no fueron sometidos a la IAFy (II) Grupo IAF, en el cual los atletas fueron sometidos a la IAF $\left(\sim 12^{\circ} \mathrm{C}\right)$ durante seis minutos. El esfuerzo físico fue realizado en una sesión de entrenamiento con simulación deluchas de 
jiu-jitsu, con cuatro luchas de cinco minutos cada una y un intervalo de tres minutos entre ellas. Se evaluaron las variables CK plasmática, protocolo estático y dinámico del Kimono Grip Strength Test y los parámetros isocinéticos (pico de par, trabajo y potencia) antes y después de los procedimientos experimentales de recuperación. Para el análisis estadístico se utilizó la prueba de varianza de dos factores (tiempo y tratamiento). El nivel de significación fue de 5\%. Resultados: Se observó el efecto del tiempo al comparar la CKplasmática (aumento de 174,39 \$99,95 UI/I para el CON y aumento de 187,91 $\pm 113,02$ UIII para la IAF) y prueba estática del KGST ( $\Delta$ t de $-5,83 \pm 9,35 \mathrm{~s}$ para CONy $\Delta t$ de $-2,83 \pm 13,94 \mathrm{~s}$ para IAF) antes y después de los procedimientos experimentales de recuperación, sin el efecto del tratamiento $(P>0,05)$. Los parámetros isocinéticos no fueron influenciados. Conclusión: Se concluye que la IAF no promovióla recuperación después del esfuerzo en atletas de juu-jitsu. Nivel de Evidencia ll; ECRC de menor calidad.

Descriptores: Creatina quinasa; Crioterapia; Artes marciales; Fuerza muscular; Rehabilitación.

\section{INTRODUÇÃO}

A recuperação pós-exercício tem sido o enfoque de diversas pesquisas. De uma maneira geral, quanto mais rápida for a recuperação, melhor é o prognóstico acerca do sistema orgânico estudado. Dentre as variáveis estudadas, destacam-se: (a) o comportamento muscular, preferencialmente utilizando testes isocinéticos; e (b) marcadores do dano muscular, como pela dosagem sanguínea da enzima Creatina Quinase (CQ).

Considerando a modalidade jiu-jitsu, deve-se destacar que esses atletas são submetidos a tarefas intermitentes e de alta intensidade, impactando inúmeros sistemas orgânicos do praticante. ${ }^{2}$ Embora seja uma prática esportiva muito utilizada no Brasil, poucos são os estudos científicos com esses atletas., ${ }^{3,4}$ Nesse sentido, é fundamental a identificação de estratégias que promovam de maneira mais eficiente a recuperação pós-treino nessa população.

De acordo com Machado et al., 5 a imersão em água fria (IAF) é uma das estratégias de recuperação pós-esforço mais utilizadas no contexto esportivo e tem sido demonstrada a redução de marcadores do dano muscular. Contudo, a literatura ainda é controversa quanto aos protocolos adotados, sobretudo com relação à temperatura da água, tempo e profundidade da imersão, ${ }^{6}$ bem como é escassa para atletas de jiu-jitsu.

Até o presente momento, foram encontrados poucos estudos envolvendo a IAF em atletas de jiu-jitsu. Fonseca et al. ${ }^{7}$ investigaram a IAF ( $\sim 6^{\circ} \mathrm{C}$, durante 19 minutos) e concluíram que a IAF pode ser benéfica em função da redução dos níveis de lactato desidrogenase (LDH), menor dor muscular e recuperação da força muscular em testes funcionais. Pinho Júnior et al. ${ }^{8}$ ao estudarem a IAF $\left(\sim 5^{\circ} \mathrm{C}\right.$ por 19 minutos), observaram que a IAF não influenciou a força em testes funcionais. Contudo, verificaram que a CQ apresentou menor elevação no grupo IAF quando comparado ao grupo controle. Santos et al. ${ }^{9}$ observaram que os efeitos agudos da IAF pós-exercício foram a redução de marcadores de dano muscular (CQ e LDH), preservação da força isométrica e redução da percepção de dor dos atletas.

Diante desse contexto, o presente estudo objetivou investigar o efeito da IAF $\left(\sim 12^{\circ} \mathrm{C}\right)$ com profundidade até o processo xifóide durante seis minutos imediatamente após um protocolo de treinamento para lutadores de jiu-jitsu. Tal proposta de protocolo se justifica no sentido de verificar uma temperatura alternativa com menor desconforto dérmico, e consequentemente maior tolerância, bem como um tempo compatível com a situação real de competição.

Para tanto, o marcador de dano muscular CQ, as variáveis isocinéticas, pico de torque, trabalho e potência muscular, bem como testes funcionais de força e preensão sugeridos no protocolo KGST (Kimono Grip Strength Tests) ${ }^{10}$ foram investigados. Foi hipotetizado que a IAF pudesse reduzir o dano muscular e favorecer a recuperação dos parâmetros isocinéticos de força muscular pós-treino.

\section{MATERIAIS E MÉTODOS}

Trata-se de um estudo do tipo cross-over com a aplicação por duas vezes de um protocolo de treinamento com simulação de lutas para atletas de jiu-jitsu, com um período de 30 dias entre as avaliações (wash-out). A amostra de conveniência foi constituída por 12 atletas de jiu-jitsu, (10 homens e 2 mulheres), com graduação azul ( $n=6)$ e amarela $(n=6)$ e com as características antropométricas apresentadas na Tabela 1. Dois grupos experimentais foram adotados: (I) Grupo Controle (CON), no qual os atletas não foram submetidos à IAF, somente recuperação passiva pós-sessão e (II) Grupo Imersão em Água Fria (IAF), no qual os atletas foram submetidos à IAF. Considerando ser um estudo cross-over, cada grupo teve a participação dos 12 atletas.

A pesquisa obedeceu aos princípios éticos para pesquisas em humanos sendo aprovada pelo Comitê de Ética em Pesquisa do Instituto Federal de Educação, Ciência e Tecnologia do Sudoeste de Minas Gerais (CAAE: 56478016.6.0000.5588). Todos os atletas assinaram o Termo de Consentimento Livre e Esclarecido (TCLE) para participarem dessa pesquisa.

Todos os procedimentos experimentais foram realizados nas dependências do Centro de Ciências Aplicadas à Educação e Saúde (CeCAES - IFSULDEMINAS, Campus Muzambinho).

Inicialmente, todos os atletas foram submetidos à uma avaliação antropométrica para obtenção dos dados apresentados na Tabela 1. No mesmo dia, os valores basais de todos os parâmetros estudados foram obtidos e, em seguida foi instituído o protocolo de treinamento com simulação de lutas de acordo com a Figura 1.

As lutas foram definidas por sorteio e com a participação de atletas com peso semelhante, com a mesma graduação da cor de faixa e mesmo sexo. Após a luta simulada, os atletas foram randomicamente alocados no grupo IAF ou CON e, imediatamente após o protocolo de recuperação, os parâmetros estudados foram novamente obtidos.

O protocolo da luta simulada obedeceu às mesmas regras da Confederação Brasileira de jiu-jitsu Esportivo, exceto para o fim da luta em função de estrangulamento ou qualquer outro tipo de finalização. Nesse caso, os atletas foram separados e imediatamente retornavam ao combate, garantindo assim o máximo de esforço físico e igualdade de tempo para todos os atletas.

Tabela 1. Características antropométricas dos atletas de jiu-jitsu.

\begin{tabular}{c|c}
\hline Variáveis & Média e desvio padrão $(\mathbf{n}=12)$ \\
\hline Peso $(\mathrm{kg})$ & $72,05 \pm 13,00$ \\
\hline Altura $(\mathrm{m})$ & $1,70 \pm 0,05$ \\
\hline Idade $(\mathrm{anos})$ & $21,75 \pm 3,10$ \\
\hline IMC $\left(\mathrm{kg} / \mathrm{m}^{2}\right)$ & $24,65 \pm 3,81$ \\
\hline Tempo de treinamento (anos) & $3,41 \pm 0,51$ \\
\hline
\end{tabular}




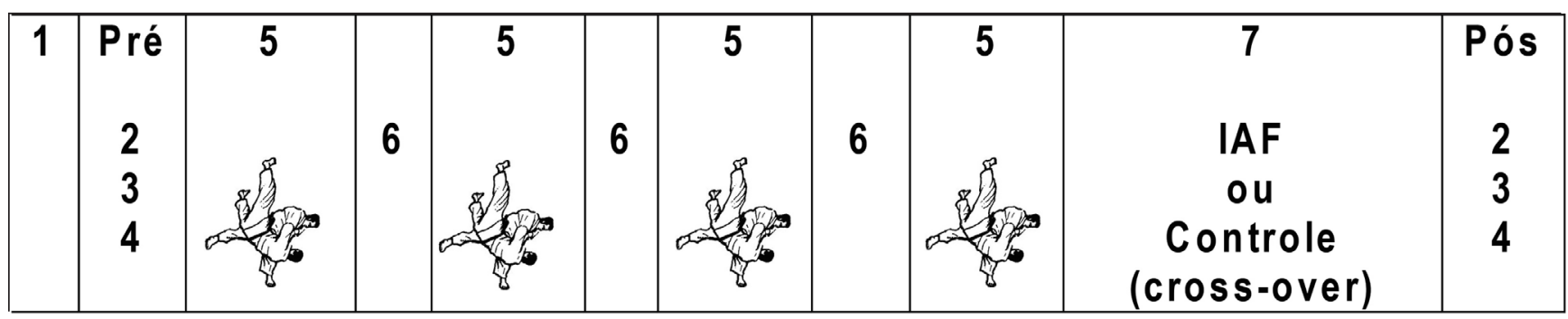

1: Antropometria; 2: Obtenção do nível sérico de creatina quinase; 3: Avaliação por meio do teste funcional (KGST) a força isométrica e dinâmica dos membros superiores; 4: Avaliação Isocinética do joelho; 5: Simulação de lutas com duração de cinco minutos; 6 : Intervalos entre a simulação de luta com duração de três minutos; 7: Recuperação em IAF (Imersão em água Fria) durante seis minutos em água com temperatura de $\sim 12^{\circ} \mathrm{C}$ ou recuperação passiva.

Figura 1. Representação esquemática de todo procedimento experimental do estudo. Os números indicam os procedimentos realizados sequencialmente.

A CQ plasmática foi avaliada por fotometria de reflectância a $37{ }^{\circ} \mathrm{C}$ com o equipamento Reflotron ${ }^{\circledR}$ Plus (Roche Diagnostics Ltd., Rotkreuz, Suíça). Para tanto, foram retirados $32 \mu \mathrm{L}$ de sangue capilar da polpa digital do dedo anelar dos atletas, após ter sido realizada limpeza do local com álcool etílico a 95\%. Em seguida, após secagem com algodão, para punção foi utilizada uma lanceta com disparador automático e o sangue foi drenado para um tubo capilar heparinizado (Cat no 955053202 Reflotron ${ }^{\circledR}$, Roche Diagnostics Ltd., Rotkreuz, Suíça). O sangue foi imediatamente pipetado para uma tira reativa de CQ (Cat no 1126695 Reflotron ${ }^{\circledR}$, Roche Diagnostics Ltd., Rotkreuz, Suiça) e colocada no equipamento para análise automática. O resultado da avaliação era demonstrado após três minutos da inserção da tira no aparelho em Unidades Internacionais por litro (UI/I). A precisão e validade desse equipamento tem sido descrita com erro inferior a 5\% comparado com técnicas laboratoriais padrão. ${ }^{11}$

Para a avaliação de força dos membros superiores foi usado o protocolo estático e dinâmico do KGST, o qual permitiu a obtenção do tempo máximo de sustentação do corpo (em segundos) e o número máximo de repetições, respectivamente. ${ }^{10}$ No protocolo estático, o atleta deveria por meio de preensão palmar, segurar um quimono que estava fixado em uma barra de metal, com os cotovelos isometricamente fletidos em aproximadamente 90 graus durante o maior tempo possível até a exaustão. No protocolo dinâmico, os atletas deveriam realizar o maior número de repetições de flexões de aproximadamente 90 graus associada à extensão máxima dos cotovelos. A ordem dos testes foi randomizada e o tempo entre os testes foi de 10 minutos. A exaustão foi determinada pela incapacidade em sustentar o corpo na posição correta para o teste estático e a incapacidade em realizar a repetição completa para o teste dinâmico. ${ }^{12}$

Para avaliação de força dos músculos quadríceps e isquiotibiais foi utilizado um equipamento de dinamometria isocinética (Biodex System Pro, Medical Systems, Nova Iorque, EUA). O atleta se posicionou sentado na cadeira do equipamento de maneira que a articulação do joelho dominante ficasse alinhada com o eixo do dinamômetro, conforme recomendação do fabricante. Por meio de feedback visual e o mesmo estímulo verbal de incentivo do pesquisador, o atleta realizava em desempenho máximo na velocidade angular de 60\%s, três séries com 10 repetições concêntricas de extensores (quadríceps) e concêntricas de flexores (isquiotibiais) de joelho. O intervalo de pausa entre as séries foi de um minuto. ${ }^{13}$ Os seguintes parâmetros isocinéticos foram registrados: Pico de Torque (PT), expresso em N/m; Trabalho Total (TT), expresso em J; e Potência Muscular (P), expresso em W.

Para o protocolo de IAF, os atletas foram alocados em uma caixa plástica contendo água fria em torno de $\sim 12^{\circ} \mathrm{C}$ e altura suficiente para atingir o processo xifóide. Essa temperatura está dentro dos parâmetros $\left(10^{\circ} \mathrm{C}\right.$ a $\left.15^{\circ} \mathrm{C}\right)$ sugeridos por Halson et al. ${ }_{1}{ }^{14}$ como adequados para promover a recuperação pós-exercício. A duração dessa imersão foi de seis minutos, uma vez que a partir desse tempo já se observa alterações autonômicas. ${ }^{15} \mathrm{O}$ atleta permaneceu sentado com as mãos apoiadas na região femoral e a temperatura da água foi monitorada com o uso de um termômetro digital infra-vermelho TD 925 (ICEL, Manaus, AM, Brasil). Caso houvesse necessidade, cubos de gelos eram adicionados a caixa plástica para o resfriamento da água e manutenção da temperatura em torno do desejável.

\section{Análise estatística}

Os dados foram expressos em média e desvio padrão (DP). Considerando que os dados apresentaram distribuição normal pelo teste Shapiro-Wilk foi adotada a Análise da Variância com dois fatores para testar a hipótese nula [ANOVA, two-way: tempo (pré versus pós) e tratamento (CON e IAF)]. O nível de significância foi de 5\% ( $P<0,05)$.

\section{RESULTADOS}

A Tabela 2 apresenta as concentrações plasmáticas médias de CQ. Foi observado efeito do tempo $\left(F_{1,44}=11,21 ; P<0,001\right)$ com menores valores na situação pré em relação à situação pós-treino. Contudo, não foi observado efeito do tratamento $\left(F_{1,44}=0,01 ; P=0,92\right)$.

A Tabela 3 apresenta os valores médios obtidos durante o KGST. Foi observado efeito do tempo $\left(F_{1,44}=4,23 ; P=0,04\right)$ com maiores valores na situação pré em relação à situação pós-treino. Contudo, não foi observado efeito do tratamento $\left(F_{1,44}=0,70 ; P=0,40\right)$ no teste estático.

Com relação ao teste dinâmico, não foram encontrados efeitos do tempo $\left(F_{1,44}=0,38 ; P=0,53\right)$ e do tratamento $\left(F_{1,44}=0,19 ; P=0,66\right)$.

A Tabela 4 apresenta os valores médios obtidos dos parâmetros isocinéticos antes e após os procedimentos experimentais. No quadríceps não foram observadas diferenças estatísticas relacionadas ao efeito do tempo: pico de torque $\left(F_{1,44}=0,52 ; P=0,47\right)$, trabalho $\left(F_{1,44}=0,54 ; P=0,46\right)$ e potência $\left(F_{1,44}=0,65 ; P=0,42\right)$. Também não foram encontradas diferenças estatísticas quanto ao efeito da IAF: pico de torque $\left(F_{1,44}=0,00 ; P=0,96\right)$, trabalho $\left(F_{1,44}=0,45 ; P=0,50\right) \mathrm{e}$ potência $\left(F_{1,44}=0,16 ; P=0,68\right)$.

Considerando os isquiotibiais, não foram observadas diferenças estatísticas relacionados ao efeito do tempo: pico de torque $\left(F_{1,44}=\right.$ $1,16 ; P=0,28)$, trabalho $\left(F_{1,44}=3,64 ; P=0,06\right)$ e potência $\left(F_{1,44}=1,84 ; P\right.$ $=0,18)$. Também não foram encontradas diferenças estatísticas quanto ao efeito da IAF: pico de torque $\left(F_{1,44}=0,35 ; P=0,55\right)$, trabalho $\left(F_{1,44}=\right.$ $3,64 ; P=0,06)$ e potência $\left(F_{1,44}=1,84 ; P=0,18\right)$.

Tabela 2. Concentrações plasmáticas de creatina quinase antes e após os procedimentos experimentais.

\begin{tabular}{c|c|c}
\hline \multirow{2}{*}{ Creatina quinase (UI/I) } & \multicolumn{2}{|c}{ Momentos experimentais } \\
\cline { 2 - 3 } & Pré & Pós \\
\hline Controle & $208,27 \pm 152,26$ & $382,66 \pm 143,02^{*}$ \\
\hline Imersão em Água Fria & $206,38 \pm 181,82$ & $394,30 \pm 252,51^{*}$ \\
\hline
\end{tabular}


Tabela 3. Valores obtidos nos testes estático e dinâmico do KGST antes e após os procedimentos experimentais.

\begin{tabular}{|c|c|c|}
\hline \multirow{2}{*}{ Testes funcionais } & \multicolumn{2}{|c|}{ Momentos experimentais } \\
\hline & Pré & Pós \\
\hline \multicolumn{3}{|l|}{ Estático (s) } \\
\hline Controle & $38,25 \pm 15,44$ & $23,75 \pm 12,51^{*}$ \\
\hline Imersão em Água Fria & $29,68 \pm 15,65$ & $26,75 \pm 14,39^{*}$ \\
\hline \multicolumn{3}{|l|}{ Teste Dinâmico (rep.) } \\
\hline Controle & $10,41 \pm 5,90$ & $8,50 \pm 6,18$ \\
\hline Imersão em Água Fria & $8,58 \pm 6,30$ & $8,25 \pm 6,81$ \\
\hline
\end{tabular}

Tabela 4. Valores obtidos dos parâmetros isocinéticos antes e após os procedimentos experimentais.

\begin{tabular}{|c|c|c|}
\hline \multirow{2}{*}{ Parâmetros isocinéticos } & \multicolumn{2}{|c|}{ Momentos experimentais } \\
\hline & Pré & Pós \\
\hline \multicolumn{3}{|l|}{ Pico de Torque (N.m) } \\
\hline \multicolumn{3}{|l|}{ Quadríceps } \\
\hline Controle & $213,20 \pm 39,76$ & $217,51 \pm 45,10$ \\
\hline Imersão em Água Fria & $227,27 \pm 47,32$ & $222,72 \pm 43,51$ \\
\hline \multicolumn{3}{|l|}{ Pico de Torque (N.m) } \\
\hline \multicolumn{3}{|l|}{ Isquiotibiais } \\
\hline Controle & $113,28 \pm 24,16$ & $107,21 \pm 22,85$ \\
\hline Imersão em Água Fria & $119,75 \pm 30,95$ & $109,63 \pm 25,89$ \\
\hline \multicolumn{3}{|l|}{ Trabalho (W) } \\
\hline \multicolumn{3}{|l|}{ Quadríceps } \\
\hline Controle & $220,27 \pm 22,58$ & $208,25 \pm 41,80$ \\
\hline Imersão em Água Fria & $208,99 \pm 42,45$ & $203,30 \pm 37,71$ \\
\hline \multicolumn{3}{|l|}{ Trabalho (W) } \\
\hline \multicolumn{3}{|l|}{ Isquiotibiais } \\
\hline Controle & $129,94 \pm 31,00$ & $117,53 \pm 28,24$ \\
\hline Imersão em Água Fria & $157,32 \pm 85,69$ & $115,01 \pm 27,70$ \\
\hline \multicolumn{3}{|l|}{ Potência (J) } \\
\hline \multicolumn{3}{|l|}{ Quadríceps } \\
\hline Controle & $144,79 \pm 27,10$ & $133,75 \pm 30,14$ \\
\hline Imersão em Água Fria & $145,43 \pm 40,23$ & $140,91 \pm 34,25$ \\
\hline \multicolumn{3}{|l|}{ Potência (J) } \\
\hline \multicolumn{3}{|l|}{ Isquiotibiais } \\
\hline Controle & $88,67 \pm 27,95$ & $77,51 \pm 20,89$ \\
\hline Imersão em Água Fria & $84,42 \pm 24,82$ & $77,23 \pm 15,12$ \\
\hline
\end{tabular}

\section{DISCUSSÃO}

O incremento de CQ plasmática tem sido associado com dano muscular e dor muscular de início tardio em atletas após o esforço físico. ${ }^{15,16}$ No presente estudo, a análise de CQ revelou que o grupo IAF não promoveu diferenças estatísticas em comparação ao grupo controle. Avaliando atletas de jiu-jitsu, Fonseca et al. ${ }^{7}$ também não encontraram diferenças nos valores de $C Q$, após a sessão de treinamento, quando compararam o grupo IAF e controle. Contudo, as investigações de Pinho-Junior et al. ${ }^{8}$ e Santos et al. ${ }^{9}$ contrastam com os achados do presente estudo, uma vez que a IAF resultou em um menor aumento da concentração de CQ após a sessão de treinamento em relação ao grupo controle.

É interessante notar que os estudos de Fonseca et al., ${ }^{7}$ Pinho-Junior et al. ${ }^{8}$ e Santos et al. ${ }^{9}$ adotaram o mesmo protocolo de IAF $\left(5^{\circ} \mathrm{C}\right.$ durante 19 minutos) e apresentaram desfechos diferentes para CQ. Contudo, observou-se que as concentrações de CQ no momento pré no estudo de Fonseca et al. ${ }^{7}$ foram muito inferiores em relação aos estudos de Pinho-Junior et al. ${ }^{8}$ e Santos et al. ${ }^{9}$ Os valores foram pelo menos duas vezes maiores (200 UI/I versus 500 UI/I) no momento pré. Considerando que no presente estudo, os atletas também apresentaram valores basais de CQ em torno de 200 UI/I, acredita-se que o efeito da IAF possa ser dependente do grau da lesão muscular. Da mesma forma, os variados achados na literatura podem ser atribuídos a diferentes protocolos experimentais e a distinta natureza dos atletas.

Outro achado dessa investigação é que após os protocolos de recuperação, a CQ estava aumentada em ambos os grupos experimentais, indicando que o esforço dos atletas de jiu-jitsu tenha sido suficiente para alterar a homeostase do tecido muscular. De maneira semelhante, esse mesmo efeito tempo (pré versus pós esforço físico) para o aumento da CQ em atletas de jiu-jitsu tem sido documentado. ${ }^{7-9}$

No presente estudo, os resultados do KGST não demonstraram diferenças estatísticas entre os grupos experimentais (IAF versus CON). Contudo, foi identificado efeito do tempo (pré versus pós) quanto da força estática. De maneira semelhante, Pinho Júnior et al. ${ }^{8}$ não encontraram diferenças entre os tratamentos mas, verificaram o efeito do tempo em atletas de jiu-jitsu altamente treinados. Por outro lado, Santos et al. ${ }^{9}$ verificaram a atenuação da redução de força estática após a IAF, algo não constatado no grupo controle. Deve-se ressaltar que, semelhante ao presente estudo, Pinho Júnior et al. ${ }^{8}$ submeteram seus voluntários à quatro lutas de sete minutos entremeadas por 15 minutos de recuperação entre elas, enquanto Santos et al. ${ }^{9}$ aplicaram uma sessão de treinamento da modalidade de 90 minutos.

Um fator que possivelmente influencia a capacidade de trabalho muscular durante o teste KGST é a eficiência neuromuscular e condicionamento físico do atleta para uma determinada tarefa. ${ }^{17-22}$ Desta forma, uma possível explicação para a queda de desempenho da força estática e não da força dinâmica, pode estar associada a reduzida capacidade de realizar trabalho estático nos atletas investigados em comparação aos trabalhos de Fonseca et al. ${ }^{7}$ e Santos et al. ${ }^{9}$ Nesses estudos, o tempo médio de trabalho estático foi cerca de 20 segundos superior ao apresentado na presente investigação.

Os resultados da literatura acerca da IAF e a recuperação muscular pós-esforço ainda permanecem controversos. Semelhante à outras intervenções, ${ }^{23-30}$ o presente estudo também verificou que a IAF não influenciou nenhum parâmetro estudado (CQ, KGST e parâmetros isocinéticos). Allan e Mawhinney ${ }^{28}$ descreveram que embora a IAF seja muito utilizada no meio esportivo com a premissa de favorecer a recuperação do atleta, essa alternativa ainda carece de comprovação científica. Também tem sido descrito que a IAF não é capaz de alterar citocinas inflamatórias, não reduz a queda exercício-induzida em testes funcionais de desempenho e muitas vezes tem efeito placebo. ${ }^{30}$

Vários fatores podem contribuir para os conflitos de resultados envolvendo a IAF. Contudo, a metodologia empregada, seja da própria IAF (temperatura, profundidade de imersão e tempo), seja dos instrumentos de avaliações e marcadores (testes funcionais, testes clínicos, marcadores bioquímicos) parecem ser os fatores críticos das divergências científicas. ${ }^{28}$ Portanto, a confiabilidade dos instrumentos utilizados deve ser levada em consideração.

Neste estudo ficou evidenciado que os parâmetros isocinéticos não foram influenciados tanto pelo fator tempo (pré versus pós-sessão de treinamento) quanto pelo fator tratamento (IAF versus controle).

Embora a relação jiu-jitsu e IAF tenha sido estudada, ${ }^{7-9}$ poucos são os estudos que utilizam o dinamômetro isocinético (padrão-ouro para a observação da força muscular). De fato, é provável que o presente estudo tenha sido o primeiro em avaliar o efeito da IAF em atletas de jiu-jitsu com esse instrumento.

Em síntese, nota-se que a IAF foi ineficaz como estratégia de recuperação pós-esforço. Contudo, também se deve considerar que (a) as lutas de jiu-jitsu simuladas causaram alguma alteração na integridade das fibras musculares de tal maneira que a CQ estava em maior concentração após o esforço físico; (b) a dinamometria e o KGST não revelaram diferenças pré versus pós, sugerindo que a provável alteração na integridade das fibras musculares dos atletas não repercutiu sobre o 
desempenho do quadríceps e isquiotibiais, bem como nas contrações dinâmicas de membro superior; (c) de alguma maneira somente as contrações isométricas foram prejudicadas pelo esforço físico.

\section{CONCLUSÃO}

Conclui-se que A IAF $\left(\sim 12^{\circ} \mathrm{C}\right)$ durante seis minutos após uma sessão de treinamento com simulação de lutas para atletas de jiu-jitsu não alterou a expressão da CQ, o desempenho nos testes funcionais de força dos membros superiores (KGST) e nenhum parâmetro isocinético (pico de torque, trabalho e potência).

\section{AGRADECIMENTO}

O autor Renato Aparecido de Souza, agradece o apoio financeiro concedido pelo Instituto Federal de Educação, Ciência eTecnologia do Sul de Minas Gerais - IFSULDEMINAS. Os autores agradecem os responsáveis pela equipe de jiu-jitsu Tatame do Bem, do IFSULDEMINAS - Campus Muzambinho, Prof. Márcio Bueno e Profa. Rafaela Rezende.

Todos os autores declararam não haver qualquer potencial conflito de interesses referente a este artigo.

CONTRIBUIÇÕES DOS AUTORES: Cada autor contribuiu individual e significativamente para o desenvolvimento do manuscrito. PRGS (0000-0002-2822-5194)*: redação do artigo, revisão e coleta dos dados; WHP (0000-0002-2070-0448)*: análise dos dados e redação do artigo; FFS (0000-0001-7739-8422)*: análise estatística e revisão do artigo; RAS (0000-0003-0125-411X): redação do artigo, revisão, coleta dos dados, análise estatística e confecção de todo o projeto de pesquisa. *ORCID (Open Researcher and Contributor ID).

\section{REFERÊNCIAS}

1. Versey NG, Halson SL, Dawson BT. Water immersion recovery for athletes: effect on exercise performance and practical recommendations. Sports Med. 2013;43(11):1101-30.

2. Ascensão A, Leite M, Rebelo AN, Magalhães S, Magalhães J. Effects of cold water immersion on the recovery of physical performance and muscle damage following an one-self soccer match. J Sports Sci. 2011;29(3):217-25.

3. Branco BH, Fukuda DH, Andreato LV, Santos JF, Esteves JV, Franchini E. The effects of hyperbaric oxygen therapy on post-training recovery in jiu-jitsu athletes. PloS One. 2016; 9-11(3):150-517.

4. Silva BV, Ide BN, Moura SM, Marocolo M, Mota GR. Neuromuscular responses to simulated brazilian jiu-jitsu fights. J Hum Kinet. 2014;(44):249-57.

5. Almeida AC, Machado AF, Albuquerque MC, Netto LM, Vanderlei FM, Vanderlei LC, et al. The effects of cold water immersion with different dosages (duration and temperature variations) on heart rate variability post-exercise recovery: a randomized controlled trial. J Sci Med Sport. 2016;19(8):676-81.

6. Minett GM, Costello JT. Specificity and context in post-exercise recovery: it is not a one-size-fits-all approach. Front Physiol. 2015;6:130

7. Fonseca LB, Brito CJ, Silva RJ, Silva-Grigoletto ME, da Silva WM Junior, Franchini E. Use of cold-water immersion to reduce muscle damage and delayed-onset muscle soreness and preserve muscle power in jiu-jitsu athletes. J Athl Train. 2016;51(7):540-9.

8. Pinho Júnior EA, Brito CJ, Santos WO, Valido CN, Mendes EL, Franchini E. Influence of cryotherapy on muscle damage markers in jiu-jitsu fighters after competition: a cross-over study. Rev Andaluza Med Deporte. 2014;7(1):7-12

9. Santos WO, Brito CJ, Pinho Junior EA, Valido CN, Mendes EL, Nunes MA, et al. Cryotherapy post-training reduces muscle damage markers in jiu-jitsu fighters. J Hum Sport Exerc. 2012;7(3):629-38

10. Franchini E, Del Vecchio FB, Matsushigue KA, Artioli GG. Physiological profiles of elite judo athletes. Sports Med. 2011;41(2):147-6.

11. Burke JJ, Fischer PM. A clinician's guide to the office measurement of cholesterol. JAMA. 1988;259(23):3444-8.

12. Silva BV, Marocolo Junior M, Simim MA, Franchini E, Mota GR. Performance in kimono grip strength tests among Brazilian Jiu Jitsu practitioners from different levels. J Comb Sports Mart Arts. 2014;5(2):11-5.

13. Lima PO, Lima AA, Coelho AC, Lima YL, Almeida GP, Bezerra MA, et al. Biomechanical differences in brazilian jiu-jitsu athletes: the role of combat style. Int J Sports Phys Ther. 2017;12(1):67-74.

14. Halson SL. Does the time frame between exercise influence the effectiveness of hydrotherapy for recovery? Int J Sports Physiol Perform. 2011;6(2):147-9

15. Bastos FN, Vanderlei LC, Nakamura FY. Effects of cold water immersion and active recovery on post-exercise heart rate variability. Int J Sports Med. 2012;33(11):873-9.

16. Koch AJ, Pereira R, Machado M. The creatine kinase response to resistance exercise. J Musculoskelet Neuronal Interact. 2014;14(1):68-77.
17. White GE, Wells GD. Cold-water immersion and other forms of cryotherapy: physiological changes potentially affecting recovery from high-intensity exercise. Extrem Physiol Med. 2013;2(1):26

18. Marinho B, Andreato LV, Follmer B, Franchini E. Comparison of body composition and physical fitness in elite and non-elite brazilian jiu-jitsu athletes. Sci Sports. 2016;31(3):129-4.

19. Báez E, Franchini E, Ramírez-Campillo R, CAÑAS-JAMETT R, HERRERA T, BURGOS-JARA C, et al. Anthropometric characteristics of top-class brazilian jiu jitsu athletes: role of fighting style. Int J Morphol. 2014;32(3):1043-50.

20. Franchini E, Takito MY, Nakamura FY, Matsushigue KA, Dal Molin Kiss MA. Tipo de recuperação após uma luta de judô e desempenho anaeróbio intermitente subseqüente. Motriz. 2001;7(1):49-52.

21. Ratamess NA. Strength and conditioning for grappling sports. Strength Cond J. 2011;33(9):35-44.

22. Oliveira M, Moreira D, Godoy JR, Cambraia AN. Evaluation of the palmar grip strength in jiu jitsu athletes in competitive level. Rev Bras Ciênc e Mov. 2006;14(3):63-70.

23. Moreira A, Costa EC, Coutts AJ, Nakamura FY, da Silva DA, Aoki MS. Cold water immersion did not accelerate recovery after a futsal match. Rev Bras Med Esporte. 2015; 21(1): 40-43.

24. Argus CK, Broatch JR, Petersen AC, Polman R, Bishop DJ, Halson S. Cold water immersion and contrast water therapy do not improve short-term recovery following resistance training. Int $J$ Sports Physiol Perform. 2016;5:1-21.

25. Yeung SS, Ting KH, Hon M, Fung NY, Choi MM, Cheng JC, et al. Yeun. Effects of cold water immersion on muscle oxygenation during repeated bouts of fatiguing exercise: a randomized controlled study. Medicine (Baltimore). 2016;95(1):e2455.

26. Jakeman JR, Macrae R, Eston R. A single 10-min bout of cold-water immersion therapy after strenuous plyometric exercise has no beneficial effect on recovery from the symptoms of exercise-induced muscle damage. Ergonomics. 2009:52(4):456-60.

27. Goodall S, Howatson G. The effects of multiple cold water immersions on indices of muscle damage. J Sports Sci Med. 2008;7(2):235-41.

28. Allan $\mathrm{R}$, Mawhinney $\mathrm{C}$. Is the ice bath finally melting? cold water immersion is no greater than active recovery upon local and systemic inflammatory cellular stress in humans. J Physiol. 2017; 595(6):1857-8

29. Roberts LA, Raastad T, Markworth JF, Figueiredo VC, Egner IM, Shield A, et al. Post-exercise cold wate immersion attenuates acute anabolic signalling and long-term adaptations in muscle to strength training. J Physiol. 2015;593(18):4285-301.

30. Peak JM, Roberts LA, Figueiredo VC, Egner I, Krog S, Aas SN, et al. The effects of cold water immersion and active recovery on inflammation and cell stress responses in human skeletal muscle after resistance exercise. J Physiol. 2017;595(3):695-711. 